\title{
Correction to: The Immediate and Long-term Effects of an Intensive Meditation Retreat
}

\author{
Jonah N. Cohen ${ }^{1} \cdot$ Dane Jensen $^{1} \cdot$ Jonathan P. Stange ${ }^{1,2} \cdot$ Mark Neuburger $^{3} \cdot$ Richard G. Heimberg $^{1}$
}

Published online: 26 July 2018

(C) Springer Science+Business Media, LLC, part of Springer Nature 2018

\section{Correction to: Mindfulness (2017) 8:1064-1077 https://doi.org/10.1007/s12671-017-0682-5}

There were two errors of note in the original manuscript:

1. On p. 1067, we stated that we used a short form of the State Trait Anxiety Inventory (STAI; Spielberger, 1983). In fact, we used the complete 20-item version of the Trait subscale of the STAI. With the exception of item 2 below, all statistics in the original manuscript relating to the full STAI-Trait are correct.

2. On p. 1071, there are errors in Table 2 regarding the STAI. All correlations in Table 2 that involve the STAI are incorrectly reported as positive. However, several negative signs were omitted, and the correlations with the following variables should all be negative (the magnitudes of all correlations are correct):
FFMQ total

FFMQ observe

FFMQ describe

FFMQ act awareness

FFMQ nonreactivity

FFMQ nonjudgment

ERQ reappraisal

CFI control

CFI alternatives

Shift costs

DAS total

These errors do not occur in similar tables provided in online supplementary materials, and the remaining correlations in Table 2 are correct. An updated version of Table 2 appears below.

The online version of the original article can be found at https://doi.org/ $10.1007 / \mathrm{s} 12671-017-0682-5$

Richard G. Heimberg

heimberg@temple.edu

1 Department of Psychology, Temple University, 1701 North 13th Street, Philadelphia, PA 19122-6085, USA

2 Department of Psychiatry, Center on Depression and Resilience, University of Illinois at Chicago, 1601 W. Taylor St.,

Chicago 60612, IL, USA

3 PRA Health Sciences, Raleigh, NC, USA 


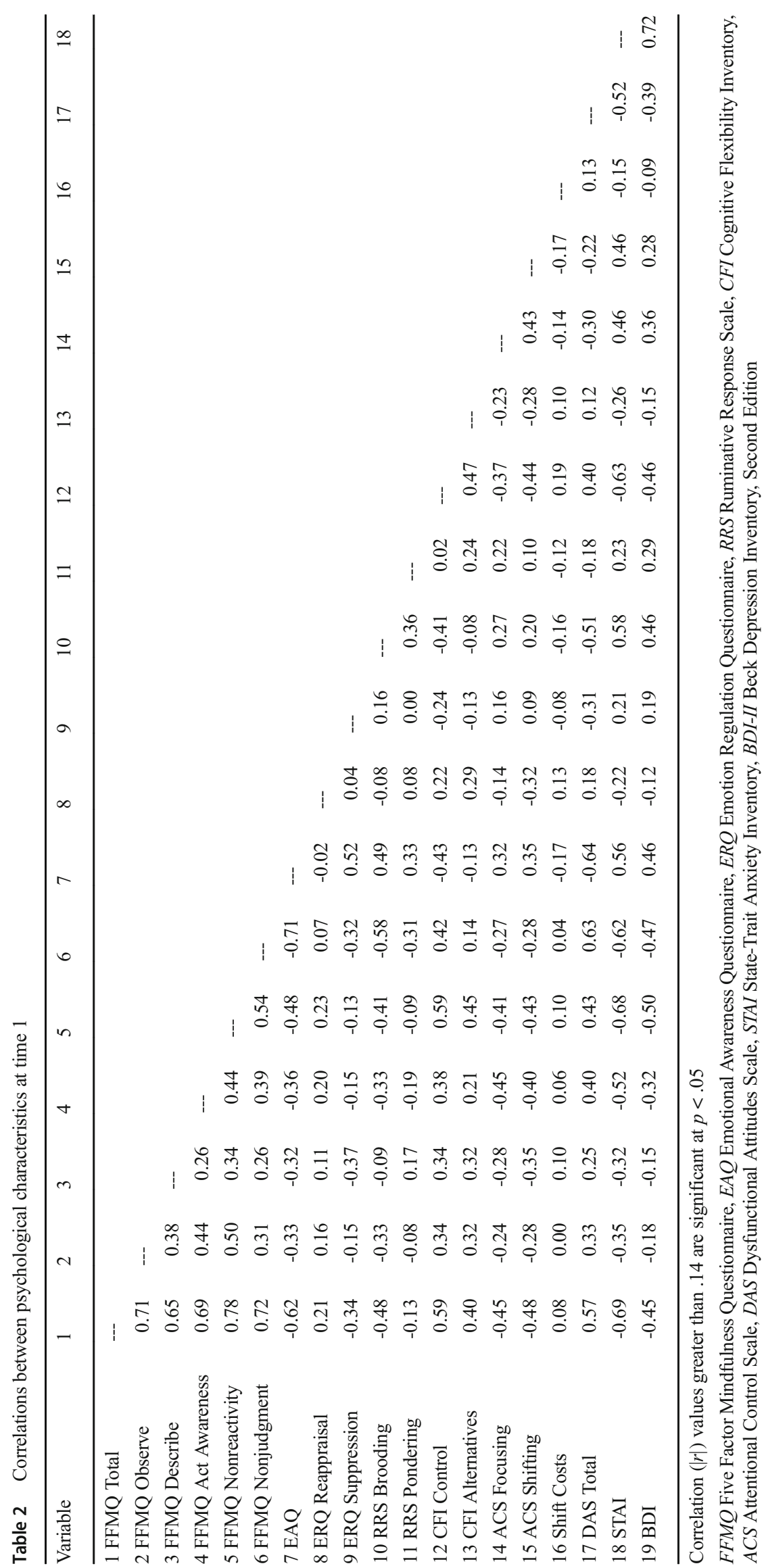

